\title{
Latent Tuberculosis Infection in Patients with Multiple Sclerosis
}

*Isabelle Pastor Bandeira1; Caroline Figueiredo da Silva1; Maria Eduarda Marchi Martin1; Gustavo Figueiredo da Silva1; Laura Fiuza Parolin² ; Luiz Henrique Melo 4; Marcus Vinícius Magno Gonçalves4

1 - Medical student - Department of Medicine, University of the Region of Joinville (UNIVILLE), Brazil.

2 - Medical Doctor and Professor of Neurology, University of the Region of Joinville (UNIVILLE), Brazil.

3 - Medical Doctor and Professor of Infectology, University of the Region of Joinville (UNIVILLE), Brazil.

4- Medical Doctor, PhD and Professor of Neurology, University of the Region of Joinville (UNIVILLE), Brazil.

\section{Corresponding Author}

Isabelle Pastor Bandeira

Department of Medicine - University of the Region of Joinville. Paulo Malschitzki, 10 - Zona Industrial Norte, CEP 89201-972, Joinville, Santa Catarina, Brazil. Phone number: +55 419 9927-8674. E-mail: isabellepbandeira@gmail.com

\section{Abstract}

Tuberculosis (TB) is an infectious-contagious disease caused by M. tuberculosis (Koch's bacillus). About one-quarter of the world's population is infected with that bacillus and at risk of developing TB disease. Latent tuberculosis corresponds to people who have been infected by TB bacteria but are not (yet) ill. The most vulnerable population to TB activation includes HIV infected, drug abuse and autoimmune disease patients.

Multiple Sclerosis (MS) is a chronic and autoimmune neurological disease caused by lymphocytic infiltration. Its prevalence worldwide is 22.2 million cases of MS. There is a relation between TB and MS: due to immunomodulation or immunosuppression treatment of MS (reactivation of latent infection), or due to the intense inflammatory response before the infection of the bacillus (increased susceptibility to the development of autoimmune diseases). Screening for TB includes complete patient history, physical exam, chest radiography, and Tuberculin Skin Test or IGRA (Interferon Gamma Release Assay).

This investigation is suggested when MS drugs (immunomodulatory and immunosuppressant medications) are prescribed. If a patient has positive results, the treatment for MS should not be delayed for the finishing TB treatment. In this paper, considering the high prevalence of tuberculosis, we recommend that TB screening should be also done at the moment of Multiple Sclerosis diagnosis.

\section{Keywords}

Multiple Sclerosis; Tuberculosis; immunosuppressive therapy; Latent Tuberculosis;

\section{Abbreviations}

TB $=$ Tuberculosis

MS = Multiple Sclerosis 
IGRA = Interferon Gamma Release Assay

$W H O=$ World Health Organization

TLRs $=$ toll-like receptors

$\mathrm{IFN} \mathrm{Y}=$ Interferon gamma

IL-17 = Interleukin-17

$B C G$ vaccine $=$ Bacillus Calmette-Guérin vaccine

$M R I=$ Magnetic resonance imaging

EAE model $=$ Experimental Autoimmune Encephalitis model

$\mathrm{LTBI}=$ latent tuberculosis infection

TST $=$ tuberculin skin test

$\mathrm{PPD}=$ purified protein derivate

$\mathrm{CXR}=$ Chest radiography

$\mathrm{CT}=$ Computed Tomography

$\mathrm{INH}=$ Isoniazid

$\mathrm{RPT}=$ Rifapentine

$\mathrm{RIF}=$ Rifampin

\section{Study funding}

No targeted funding reported.

\section{Disclosure}

The authors report no disclosures. All authors and contributors agree to the conditions outlined in the Authorship and Contributorship section of the Information for Authors. The authors have read the Journal's position on issues involved in ethical publication. There was no sponsorship for the scientific article and there was no conflict of interest with all the authors. 


\section{MANUSCRIPT}

\section{Background:}

Tuberculosis (TB) is an infectious-contagious disease caused by M. tuberculosis (Koch's bacillus) that most often affects the lungs. For the past 25 years, it has has been considered a global public health emergency: continues to cause considerable morbidity and mortality globally (Furin $\mathrm{J}$ et al. 2019). In 2015, there were 10.4 million new TB cases and about 1.4 million deaths (Reis Santos, 2019). According to WHO, about one-quarter of the world's population is infected with $M$. tuberculosis and thus at risk of developing TB disease (WHO 2019).

The effect of poverty on the incidence of TB is unassailable. Underdeveloping countries such as Brazil, the Russian Federation, India, China and South Africa (BRICS) together account for approximately $50 \%$ of tuberculosis cases worldwide. In 2010, 68,729 new cases of pulmonary TB were reported in Brazil, with an incidence rate of 36 cases per 100,000 inhabitants (Reis-Santos B., et al. 2019; Zille A, et al. 2019). Therefore, tuberculosis infection remains an important public health problem in Brazil. The most vulnerable population to TB are homeless persons, prisoners, health professionals, and indigenous populations (Malacarne J, et al. 2016).

Latent tuberculosis means people who have been infected by TB bacteria but are not (yet) ill with the disease and cannot transmit the disease (WHO 2019). The risk factors for disease activation are HIV infection, malnutrition, smoking, alcohol abuse, drugs, diabetes, lung and autoimmune diseases such as multiple sclerosis (MS) (Lönnroth K, et al. 2009).

Multiple Sclerosis is a chronic and autoimmune neurological disease characterized by demyelination, multifocal inflammation, reactive gliosis and axonal losses, caused by lymphocytic infiltration, which courses with a high disability of the patient (Zettl $U$, et al. 2012). The prevalence of this disease continues to increase: in 2016, there were about 22.2 million cases of multiple sclerosis in the world, with around 30,000 cases in Brazil. (Wallin M, et al. 2016).

MS treatment consists of immunological modulation or suppression, to contain the intense inflammatory process that occurs at these patients (Fragoso Y, et al. 2014). Thus, patients with MS are subjected to new infections, reactivation of latent pathogens and worsening of asymptomatic chronic infections (Epstein D, et al 2018) what is a common cause of comorbidity and death at these patients (Marrie R, et al. 2014).

\section{Tuberculosis and Multiple Sclerosis relation}

The relation between TB and MS is twofold. First, due to immunomodulation or immunosuppression treatment, reactivation of latent infection may occur; second, due to the intense inflammatory response of the organism before the infection of the bacillus, there may be an increase in the susceptibility to the development of autoimmune diseases, such as MS. This one occurs because it has been observed a homology between epitopes from the chaperone HSP60 of $M$. tuberculosis and fragments of HSP60 from patients with MS. In specially, one peptide of the bacillus binds to many alleles with high affinity, this cross-reactive epitopes can be involved at the pathogenesis of MS, as it induces a high immunological response (Fragoso Y, et al. 2014)

Cossu D and colleagues 2017 demonstrated a relationship between mycobacteria and MS. Mycobacterial components are potent activators of the innate immune system via toll-like receptors (TLRs). The stimulation of the host immune response with TLR2 and TLR4 induces the production of cytokines. These induce the differentiation of naive CD4 + T cells into Th1 and Th17 cells, and the consequent production of IFNy and IL-17. The whole set facilitates leukocyte transmigration across 
the blood-brain-barrier, contributing to tissue damage and neuronal dysfunction in MS. (Cossu D, et al. 2017)

The relationship between the BCG vaccine and multiple sclerosis appears to be beneficial. In 1999, G. Ristori observed a 57\% reduction in Magnetic resonance imaging (MRI) activity in a cohort of 12 unselected patients with relapsing-remitting MS after a single BCG vaccination (Ristori G, et al. 1999). Similar positive results appear in the experimental autoimmune encephalitis (EAE) model. Nevertheless, it is not yet possible to affirm the real protective effect of BCG - further studies in the area are needed (Fragoso Y, et al. 2014).

\section{Which MS patients are screened for Latent Tuberculosis?}

The screening for TB is suggested when MS drugs (immunomodulatory and immunosuppressant medications) are prescribed. The reason is the high prevalence of TB infection and the impact of MS treatments on the immune system. Active TB disease in adults typically results from reactivation of latent TB (Epstein D, et al 2018). Identifying patients with a high risk of TB, such as patients from countries of high TB incidence and patients in risk factor groups, is of great importance. (Epstein D, et al 2018).

The treatment of MS involves modulation of the immune system with interferons, glatiramer acetate and immunosuppressive medications. The group of immunosuppressive medications is formed by monoclonal antibodies (natalizumab, alemtuzumab and ocrelizumab), a chemotherapeutic agent (mitoxantrone) and small-molecule oral agents (fingolimod, dimethyl fumarate, teriflunomide and daclizumab) (Epstein D, et al 2018). Each aforementioned medication has its own mechanisms of action but, as immunosuppressive drugs, all of them carry the risks of opportunistic infections like latent tuberculosis infection (LTBI).

Screening for LTBI should be prioritized for patients receiving Alemtuzumab, a humanized monoclonal antibody targeting CD52. The usage of this medication results in prolonged and intense lymphocytopenia (Epstein D, et al 2018). Due to its effect on humoral and cell-mediated immunity, Alemtuzumab shows a very high risk of reactivating tuberculosis and other opportunistic infection. Do not prescribe it if there is a risk of tuberculosis for your patient. (Fragoso Y, et al. 2014) LTBI screening is also recommended for Teriflunomide. This drug is a dihydroorota deydrogense inhibitor that affects lymphocyte proliferation and has potential chances of TB reactivation (Epstein D, et al 2018).

In contrast, ocrelizumab is likely not associated with reactivation of TB. The explanation involves the fact that these drugs have specificity for CD20, bringing a depletion of B-cell without affecting cellmediated immunity. Screening for LTBI before use anti-CD20 monoclonal antibodies, like ocrelizumab or rituximab, is not routine (Epstein D, et al 2018). There are no associations of tuberculosis during the use of Interferon- Beta or Glatiramer Acetate, which are drugs used for treating MS (Fragoso Y, et al. 2014).

\section{Diagnosing \& treating tuberculosis in MS patients}


The tuberculin skin test (TST), also known as purified protein derivate (PPD) or tuberculin test, is the first choice for the diagnosis of latent TB and have a sensitivity of $75 \%$ for patients not vaccinated with Bacillus Calmette- Guérin (BCG) and $59 \%$ in vaccinated patients and a specificity of $75 \%$. This test is considered positive when its transverse diameter, measured after $72 \mathrm{~h}$, is $>=10 \mathrm{~mm}$ in immunocompetent patients and $>=5 \mathrm{~mm}$ in immunocompromised. It is important to know that a negative TST does not exclude latent TB at any age and especially in immunocompromised patients. (Navas C, et al. 2018)

The IGRA (Interferon Gamma Release Assay) tests detect the release of interferon-gamma in response to specific antigens for TB. IGRAs have better specificity than the TST tests and are not affected by vaccination under normal conditions. However, the IGRA tests are not the first choice in the reason for the higher cost and lower availability. (Navas C, et al. 2018). In addition, multiple sclerosis-modifying therapies that affect lymphocytes have a higher risk of indeterminate IGRA tests. (Baldassari L, et al. 2019) Screening for LTBI also involves a complete history and physical exam and chest radiography to rule out active infection before initiating treatment for $L T B I$, as also for MS. (Epstein et al. 2018)

Chest radiography (CXR), is a rapid imaging technique that allows lung abnormalities to be identified. CXR has a high sensitivity for pulmonary TB and is an important tool for triaging pulmonary TB, especially when pulmonary TB cannot be confirmed bacteriologically. In case of tuberculosis, it can be seen apical fibronodular lesions, calcified solitary nodules, calcified lymph nodes, or pleural thickening in the images. Chest computed tomography (CT) scans could be complementary and provide additional information. (WHO 2016; Guirao-Arrabal E, et al. 2018).

If a patient has positive results, the treatment for MS should not be delayed for the finishing TB treatment. The treatment of latent tuberculosis can be: a) 3 months of once-weekly Isoniazid (INH) $15 \mathrm{mg} / \mathrm{kg}$ - 900mg max. plus Rifapentine (RPT) $\geq 50.0 \mathrm{~kg}, 900 \mathrm{mg}$ maximum; b) 4 months of daily Rifampin (RIF) $10 \mathrm{mg} / \mathrm{kg}$ - 600mg max.; c) 3 months of daily Isoniazid (INH) $5 \mathrm{mg} / \mathrm{kg}-300 \mathrm{mg}$ max. plus Rifampin (RIF) $10 \mathrm{mg} / \mathrm{kg}-600 \mathrm{mg}$ max. These options have made the treatment effective, safe and, as it is for a short period of time, there is greater patient compliance. Alternative therapies regimens are daily Isoniazid for 6 or 9 months, which is efficacious but has higher hepatotoxic toxicity and lower adherence. There is a potential risk of treatment-related hepatotoxicity with antiTB and MS treatments. Thus, monitoring of liver enzyme values with consideration of age as well as comorbid conditions like alcoholism, obesity, hepatotoxic drugs and liver disease should be done. (Sterling T, et al. 2020; Navas C, et al. 2018).

\section{Conclusions}

Considering the high prevalence of tuberculosis worldwide, we hardly recommend that TB screening should be carried out at the time of Multiple Sclerosis diagnosis and before starting MS immunomodulatory therapy. The sooner the presence of the $M$. tuberculosis bacillus is identified and treated, the better is the patient's prognosis. When correctly managed, around 60 to $90 \%$ of cases do not develop the active form (Kim H, et al 2018).

Tuberculosis screening in Multiple Sclerosis patients includes epidemiological risk assessment, chest radiography and bacteriological tests (TST/PPD and/or IGRA) annually. For epidemiological evaluation, the local epidemiology of the disease must be taken into account, as well as contact with a bacilliferous patient in household conditions (did they share a bedroom? What was the exposure time? Was the place ventilated?). A negative contact history does not exclude LTBI. 


\section{Summary}

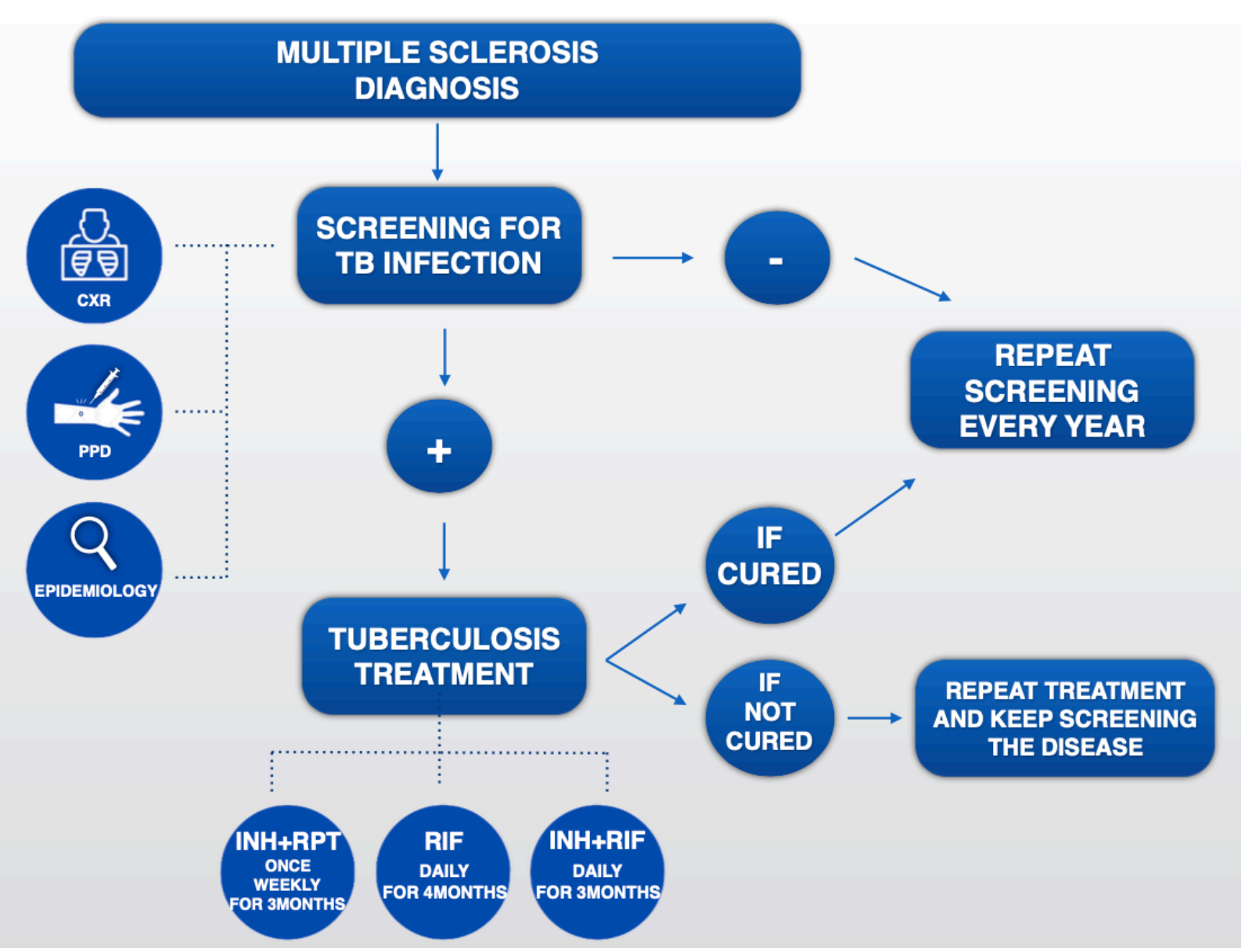

Figure 1: Summary of Tuberculosis screening that should be carried out at the time of Multiple Sclerosis of diagnosis and before starting MS immunomodulatory therapy. 


\section{REFERENCES:}

Baldassari L, Feng J, Macaron G, Planchon S, Alshehri E, Moss B et al. Tuberculosis screening in multiple sclerosis: effect of disease-modifying therapies and lymphopenia on the prevalence of indeterminate TB screening results in the clinical setting. Multiple Sclerosis Journal - Experimental, Translational and Clinical. 2019;5(3):205521731987546.

Cossu D, Yokoyama K, Hattori N. Conflicting Role of Mycobacterium Species in Multiple Sclerosis. Frontiers in Neurology. 2017;8.

Epstein D, Dunn J, Deresinski S. Infectious Complications of Multiple Sclerosis Therapies: Implications for Screening, Prophylaxis, and Management. Open Forum Infectious Diseases. 2018;5(8).

Fragoso Y, Adoni T, Anacleto A, Brooks J, Carvalho M, Claudino R et al. How do we manage and treat a patient with multiple sclerosis at risk of tuberculosis?. Expert Review of Neurotherapeutics. 2014;14(11):1251-1260.

Furin J, Cox H, Pai M. Tuberculosis. The Lancet. 2019;393(10181):1642-1656.

Guirao-Arrabal E, Torre-Cisneros J. Tuberculin skin test, Interferon gamma release assays or just chest x-ray to study latent tuberculosis before solid organ transplantation?. Transplant Infectious Disease. 2018;20(4):e12920.

Kim H, Kim J. Treatment of Latent Tuberculosis Infection and Its Clinical Efficacy. Tuberculosis and Respiratory Diseases. 2018;81(1):6.

Lönnroth K, Jaramillo E, Williams B, Dye C, Raviglione M. Drivers of tuberculosis epidemics: The role of risk factors and social determinants. Social Science \& Medicine. 2009;68(12):2240-2246.

Malacarne J, Rios D, Silva C, Braga J, Camacho L, Basta P. Prevalence and factors associated with latent tuberculosis infection in an indigenous population in the Brazilian Amazon. Revista da Sociedade Brasileira de Medicina Tropical. 2016;49(4):456-464.

Marrie R, Bernstein C, Peschken C, Hitchon C, Chen H, Fransoo R et al. Intensive care unit admission in multiple sclerosis: Increased incidence and increased mortality. Neurology. 2014;82(23):2112-2119.

Navas C, Torres-Duque C, Munoz-Ceron J, Álvarez C, García J, Zarco L et al. Diagnosis and treatment of latent tuberculosis in patients with multiple sclerosis, expert consensus. On behalf of the Colombian Association of Neurology, Committee of Multiple Sclerosis. Multiple Sclerosis Journal - Experimental, Translational and Clinical. 2018;4(1):205521731775220.

Reis-Santos B, Shete P, Bertolde A, Sales C, Sanchez M, Arakaki-Sanchez D et al. Tuberculosis in Brazil and cash transfer programs: A longitudinal database study of the effect of cash transfer on cure rates. PLOS ONE. 2019;14(2):e0212617.

Ristori G, Buzzi M, Sabatini U, Giugni E, Bastianello S, Viselli F et al. Use of Bacille Calmette-Guerin (BCG) in multiple sclerosis. Neurology. 1999;53(7):1588-1588.

Sterling T, Njie G, Zenner D, Cohn D, Reves R, Ahmed A et al. Guidelines for the Treatment of Latent Tuberculosis Infection: Recommendations from the National Tuberculosis Controllers Association and CDC, 2020. MMWR Recommendations and Reports. 2020;69(1):1-11.

Wallin M, Culpepper W, Nichols E, Bhutta Z, Gebrehiwot T, Hay S et al. Global, regional, and national burden of multiple sclerosis 1990-2016: a systematic analysis for the Global Burden of Disease Study 2016. The Lancet Neurology. 2019;18(3):269-285.

World Health Organization. Chest radiography in tuberculosis detection - summary of current WHO recommendations and guidance on programmatic approaches. WHO (2016). 36p.

World Health Organization. Global tuberculosis report. WHO, Geneva (2019). 
Zettl U, Stüve O, Patejdl R. Immune-mediated CNS diseases: A review on nosological classification and clinical features. Autoimmunity Reviews. 2012;11(3):167-173.

Zille A, Werneck G, Luiz R, Conde M. Social determinants of pulmonary tuberculosis in Brazil: an ecological study. BMC Pulmonary Medicine. 2019;19(1). 